\title{
The Viterbi Decoding Complexity of Linear Block Codes ${ }^{1}$
}

Robert J. McEliece

Californis Institute of Technology

Pasadena, California 91125, USA

Abstract - A given linear block code can be represented by many different trellises. In this paper, we will show that if these trellises are scored according to the complexity of implementing the Viterbi decoding algorithm on them, there is a uniquely optimal one, viz. the "Wolf-Massey-Muder" trellis. We will also introduce "minimal-span" generator matrices, which permit easy construction of WMM trellises.

I. Introduction. Problem Statement.

We are interested in soft-decision decoding algorithms for linear block codes, and of course a trellis for a block code can be used to implement soft-decision Viterbi decoding for the code. However, a block code can be represented by many different trellises. In order to compare different trellises, we give a standardized definition broad enough to include all the trellises devised so far: A trellis for an $(n, k)$ binary linear code $\mathcal{C}$ is a finite directed graph, in which every vertex (a.k.a. state) is assigned a "depth" in the range $\{0,1, \ldots, n\}$, and each edge must connoct a vertex at depth $i$ to one at depth $i+1$, for $i=0,1, \ldots, n-1$. There is only one vertex at depth 0 , called $A$, and only one at depth $n$, called $B$. Each edge is labelled either " 0 " or " 1 ". It is required that there be exactly $2^{k}$ directed paths from $A$ to $B$, and the labels on these paths correspond, one-to-one, with the codewords in $C$.

If we view a trellis as a blueprint for Viterbi decoding of the code, it is easy to see that decoding one received word requires $|E|$ floating-point additions, and $|E|-|V|+1$ binary comparisons, where $E$ is the set of edges in the trellis, and $V$ is the set of vertices. Thus it is natural to seek the trellis or trellises for $\mathcal{C}$ for which $|E|$ and/or $|E|-|V|$ are as small as possible.

\section{The Viterai Decoding Optimality of the} WOLF-MASSEY-MUder TrELlis.

There is, of course, one "canonical" trellis associsted with each linear code, vir. the unique "minimal trellis" discussed by Muder [4]. In fact, Kot and Leung [5] have shown that the minimal trellis is isomorphic to both the original code trellis introduced by Wolf [1], and to the trellis introduced shortly afterward by Massey [2]. However, the term "minimal" in "minimal trellis" refers to the property of minimizing the number of vertices at each depth in the trellis, not the Viterbi decoding complexity, and so prefer the term "WMM trellis" (for Wolf-Massey-Muder) for this important structure.

We can show that the WMM trellis uniquely minimizes the quantity $|E|$. The problem of minimizing $|E|-|V|$ is more difficult, but we can show that the WMM trellis minimizes $|E|-|V|$ among all linear trellises for the code. (A linear

${ }^{1}$ This work was partially supported by AFOSR grant no. F49620-94-1-005, and by grant from Pacific Bell. A portion of the work was also done at the Jet Propulsion Laboratory, Californis Institute of Technology, under contract to the National Aeronautics and Space Administration. trellis is one for which the state of a codeword $\mathcal{C}$ at time $i$ is a linear function of of $\mathcal{C}$.) The problem is more difficult than that of minimizing $|E|$ or $|V|$, because for $|E|-|V|$, the minimum is often attained for several different trellises.

To compute $|E|$ and $|V|$ for the WMM trellis, we can use the fact that the number of vertices at depth $i$ in the WMM trellis is $2^{k-p_{i}-f_{i}}$, and the number of edges connecting vertices at depth $i$ to vertices at depth $i+1$ is $2^{k-p_{i}-f i+1}$, where $p_{i}$ and $f_{i}$ are the dimensions of the ith "past" and "future" subcodes of $\mathcal{C}$, defined in [3] and [4]. In the next section we will outline an efficient procedure for computing the numbers $p_{i}$ and $f_{i}$ for any linear code. With slight elaboration, it also provides the best way of constructing the WMM trellis.

III. Minimal-Span Generator Matrices.

If $x=\left(x_{1}, \ldots, x_{n}\right)$ is a binary $n$-vector, define its support, abbreviated Supp $x$, as the smallest interval $[i, j]=\{i, i+$ $1, \ldots, j\}$, which contains all the nonzero components of $x$. Similarly, the span of $x$, denoted $\operatorname{span} x$, is the number of elements in the support of $x$. If $G$ is a $k \times n$ binary matrix, its span is defined to be the sum of the spans of its rows. We then define a minimal span genenator matrix (MSGM) for an $(n, k)$ binary linear code $\mathcal{C}$ to be a generator matrix for $\mathcal{C}$ for which the span is a minimum. Here are some of the properties of MSGM's :

-A MSGM can be found by a "greedy" algorithm: If there is an elementary row operation that can reduce the span of one of the rows of $G$, this operation should be performed. If no such operation exists, the matrix has minimal span.

-A MSGM is minimal in a very strong sense: If $G$ is a MSGM for $\mathcal{C}$, and if $G^{\prime}$ is any other generator matrix for $\mathcal{C}$, then it is possible to reorder the rows of $G^{\prime}$ so that Supp $g_{i} \subseteq$ Supp $g_{i}^{\prime}$ for $i=1, \ldots, k$, where $\left(g_{1}, \ldots, g_{k}\right)$ are the rows of $G$ and $\left(g_{1}^{\prime}, \ldots, g_{k}^{\prime}\right)$ are the rows of $G^{\prime}$.

-A MSGM is what Forney [3] calls "trellis oriented," i.e., the dimensions $p_{i}$ and $f_{i}$ of the past and future subcodes can be read directly from the matrix. $p_{i}$ is the number of rows of $G$ for which the rightmost nonzero entry lies in column $i$ or earlier, and $f_{i}$ is the number of rows of $G$ for which the leftmost nonzero entry lies in column $i+1$ or greater.

\section{REFERENCES}

[1] J. K. Wolf, "Efficient maximum likelihood decoding of linear block codes using a trellis." IEEE Trans. Inform. Theory, vol. IT-24 (1978), pp. 76-80.

[2] J. L. Masey, "Foundations and methods of channel coding." Proc. Int. Conf. Inform. Theory and Systems, NTGFachberichte vol. 65 (1978), pp. 148-157.

[3] G. D. Forney, Jr., "Coset codes - Part II: Binary lattices and related codes." IEEE Trans. Inform. Theory, vol. IT-34 (1988), pp. 1152-1187.

[4] D. J. Muder, "Minimal trellises for block codes." IEEE Trans. Inform. Theory, vol. IT-43 (1988), pp. 1049-1053.

[5] A. D. Kot and C. Leung, "On the construction and dimensionality of linear block code trellises." Proc. 1993 ISIT, p. 291. 